\title{
Perforación Optimizada de Pozos con un Taladro de 1000 hp en los Campos PBHI-MDC
}

\author{
Landívar, Rodolfo'; Loaiza, Marco²; Valencia, Raul ${ }^{3}$ \\ ${ }^{1}$ Schlumberger del Ecuador S.A., Servicios Integrados de Perforación, Quito, Ecuador \\ ${ }^{2}$ Enap Sipec, Ingeniería de Perforación, Quito, Ecuador \\ ${ }^{3}$ Escuela Politécnica Nacional, Facultad de Ingeniería en Geología y Petróleos, Quito, Ecuador
}

\begin{abstract}
Resumen: Los campos donde se realizó el estudio se localizan en la Cuenca Oriente de Ecuador, donde se perforaron pozos hasta el año 2015 con taladros de mínimo 1500 HP; para el año 2016 la Empresa Operadora tomó el desafío de optimizar los costos de perforación, evaluándose la opción de usar un equipo de menor capacidad, seleccionándose un taladro de $1000 \mathrm{HP}$, que presentaba desventajas en capacidades de torque, levantamiento de cargas, capacidad de mesas y equipos en general comparados con un taladro de $2000 \mathrm{HP}$, pero mediante la revisión de los pozos perforados previamente, planificación efectiva y eficiente, diseño de pozos acordes al equipo de perforación, generación de una matriz detallada de riesgos de perforación con acciones de prevención y mitigación, complementada con una gestión operativa enfocada en la identificación de riesgos y de oportunidades de mejora continua, en complemento la aplicación de tecnologías de punta, permitieron la viabilidad del uso de este equipo. Hasta diciembre del 2017, se perforaron 4 pozos direccionales tipo "J" modificado hasta profundidades promedio de 10882 pies, logrando ahorros en costo y tiempo, validándose la perforación con un taladro de 1000 HP.
\end{abstract}

Palabras clave: Gestión, optimización, perforación, planificación, riesgos, Taladro.

\section{Optimized Well Drilling with a 1000 HP Rig in the PBHI-MDC Fields}

\begin{abstract}
The fields where the present work was carried out are located in the Oriente Basin of Ecuador, where wells were drilled up to 2015 with at least 1500 HP Rigs; for 2016 the Operating Company took the challenge of optimizing drilling costs, evaluating the option of using a lower capacity Rig, selecting a 1000 HP Rig, which presented disadvantages in torque capacities, lifting loads, capacity of tables and equipment in general compared to a $2000 \mathrm{HP}$ Rig, but through the analysis of previously drilled wells, effective and efficient planning, design of wells according to the drilling equipment, generation of a detailed risk matrix of drilling activities with prevention and mitigation actions, complemented by an operational management focused on the identification of risks and opportunities for continuous improvement, in addition to the application of state-of-the-art technologies, allowed the viability of the use of this equipment. Up to December 2017, 4 modified "J" directional wells were drilled to an average depth of 10,882 feet, achieving cost and time savings, validating the drilling with a $1000 \mathrm{HP}$ drill.
\end{abstract}

Keywords: Management, optimization, drilling, planning, risks, rig.

\section{INTRODUCCIÓN}

El taladro de perforación es un servicio costoso durante la fase de perforación de pozos, por lo que minimizar costos es clave para optimizar los presupuestos de las empresas Operadoras de Campos Petroleros.

En el 2016 se evaluó la opción de usar un taladro de menor capacidad al de 2000 HP que estaba siendo empleado en los campos analizados en este trabajo, en pozos con profundidades de perforación promedias de 10500 pies, con desplazamientos horizontales de $3300 \mathrm{ft}$ hasta la formación Hollín, sin afectar a las personas, la integridad operacional ni al ambiente y que genere un valor agregado en disminución de costos.
No se ha encontrado antecedentes de perforación de pozos direccionales a las profundidades mencionadas con taladros de menos de $1500 \mathrm{HP}$ en el Ecuador. El taladro operativo y disponible en Ecuador con capacidad por debajo de $1000 \mathrm{HP}$ es el Tuscany 102 de 1000 HP, por lo que se enfocó el análisis en este equipo.

El estudio se realizó a 4 pozos perforados en la Cuenca Oriente del Ecuador entre el 2016 y 2017, con el taladro de perforación de $1000 \mathrm{HP}$. Las ventajas y desventajas de un taladro de $1000 \mathrm{HP}$ en comparación a uno de 2000 HP son: 
Ventajas

- Movilización y armado rápidos ( 7 días)

- Requiere menos espacio en locación

- Menor número de cargas a ser movilizadas que implica menor probabilidad de accidentes

- La torre es auto transportada

- Los costos diarios de operación de perforación son menores

- Los costos de movilización son menores al tener menos cargas de transporte

- El consumo de energía es menor

- Menor consumo de diésel implica ahorro de 1000 USD/d (300 gal/d menos)

- Se puede completar los pozos con el mismo taladro con menores tarifas que un taladro convencional de perforación

Desventajas

Capacidad limitada de trabajo y margen de maniobra de un taladro de $1000 \mathrm{HP}$ versus uno de $2000 \mathrm{HP}$, para el escenario de los pozos a perforarse, como se muestra en la tabla 1 (Tuscany, RIG 102 SPECS and Inventory Perforacion.pdf, 2018; Tuscany, RIG 117 SPECS and inventory.pdf, 2018).

Tabla 1. Especificaciones de un Taladro de $1000 \mathrm{HP}$ vs $2000 \mathrm{HP}$

\begin{tabular}{|c|c|c|}
\hline Ítem & Capacidad Nominal & Capacidad Nominal \\
\hline Potencia de Malacate & $1000 \mathrm{HP}$ & $2000 \mathrm{HP}$ \\
\hline Capacidad de Malacate & $410000 \mathrm{lb}$ & 1000000 \\
\hline Mástil & $410000 \mathrm{lb}$ & $1000000 \mathrm{lb}$ \\
\hline Capacidad Rotativa & $410000 \mathrm{lb}$ & $1000000 \mathrm{lb}$ \\
\hline $\begin{array}{c}\text { Capacidad de } \\
\text { Almacenaje de Tubería } \\
\text { (Set Back) }\end{array}$ & $250000 \mathrm{lb}$ & $488120 \mathrm{lb}$ \\
\hline $\begin{array}{l}\text { Capacidad Total } \\
\text { Simultanea }\end{array}$ & $660000 \mathrm{lb}$ & $1488120 \mathrm{lb}$ \\
\hline Top drive & $\begin{array}{c}30260(80 \mathrm{rpm}) \\
15130(160 \mathrm{rpm})\end{array}$ & $\begin{array}{l}42700(130 \mathrm{rpm}) \\
22800(190 \mathrm{rpm})\end{array}$ \\
\hline Bombas & 3 de $1000 \mathrm{HP}$ & 3 de $1600 \mathrm{HP}$ \\
\hline Tanques & $\begin{array}{l}\text { Sistema activo } \\
\quad 750 \mathrm{bls} \\
\text { Pildorero de } 80 \mathrm{bls}\end{array}$ & $\begin{array}{c}\text { Sistema activo } 1300 \mathrm{bls} \\
\text { Pildorero de } 112 \mathrm{bls}\end{array}$ \\
\hline Altura de la Torre & $114 \mathrm{ft}$ & $150 \mathrm{ft}$ \\
\hline $\begin{array}{l}\text { Número de Juntas por } \\
\text { parada }\end{array}$ & 2 & 3 \\
\hline Altura de la Mesa & $17.5 \mathrm{ft}$ & $29.5 \mathrm{ft}$ \\
\hline
\end{tabular}

- Aumenta el riesgo en las operaciones rutinarias de manejo de tubulares ya que al ser más pequeña la mesa del taladro dificulta el armado de ensamblajes de fondo y corrida de revestidores.

- Perforación más lenta ya que las paradas son solo de 2 juntas por la altura de la torre (114 ft), mientras que en una torre de $2000 \mathrm{HP}$ se pueden armar paradas de 3 juntas, esto implica mayor número de tiempo empleado en conexiones y más tiempo de exposición del hoyo.

- Dada la capacidad de la subestructura de perforación solo se pueden colocar 8000 pies de tubería de perforación sobre la misma, implicando tiempos extras en armar tubulares de caballetes cuando se viaja dentro del hoyo o quebrarlos cuando se sacan los mismos.

- Mayores tiempos de corrida de revestidor por el espacio en la mesa de perforación.

Entendido el problema del presente estudio, se plantea la pregunta: ¿Es posible perforar un pozo con un taladro de 1000 HP hasta la formación Hollín en profundidades promedios de 10500 pies? La respuesta a la interrogante permite la validación de la viabilidad del uso de un taladro de perforación de 1000 HP.

Se planteó el objetivo general de: “Optimizar la perforación de pozos para la formación Hollín con un taladro de 1000 HP" con los siguientes objetivos específicos:

- Determinar experiencias previas de perforaciones con taladros de 1000 HP o menos en el Ecuador

- Recolectar y validar los datos de perforación de los pozos perforados por La Empresa Operadora.

- Revisar del estado del arte, tecnologías, productos, herramientas y programas de perforación

- Evaluar los parámetros de perforación óptimos en relación a los límites del taladro de 1000 HP de los pozos perforados por La Empresa Operadora

- Determinar los riesgos de perforar con una torre de 1000 HP en relación a una de mayor capacidad

- Examinar la viabilidad de continuar perforando con torres de $1000 \mathrm{HP}$ en La Empresa Operadora y en otros campos de la Cuenca Oriente del Ecuador

- Determinar el límite técnico para perforar pozos con un taladro de $1000 \mathrm{HP}$

\section{MATERIALES Y MÉTODOS}

La metodología se fundamentó en el manejo de los riesgos mediante su identificación, implementando barreras para que no se materialicen e incorporando medidas de control para evitar escalamientos, en complemento una planificación realizada con suficiente tiempo para cubrir los requerimientos de personal, herramientas y servicios necesarios, resultado en un programa de perforación que recoja no solo las operaciones planeadas si no los planes de optimización a implementarse, continuando con una ejecución de las operaciones con prácticas operacionales ajustadas a los pozos específicos y limitaciones del taladro, a más del uso de tecnologías de punta, programas especializados de ingeniería y personal de experiencia trabajando en equipo para lograr la sinergia necesaria para alcanzar los objetivos del pozo.

Los servicios de perforación en los pozos perforados se manejaron a través de la compañía proveedora de los Servicios Integrados de Perforación (IDS, por sus siglas en inglés) trabajando en equipo con el personal técnico de la Empresa Operadora y de la compañía proveedora del taladro de perforación. 


\subsection{Optimización de la Perforación}

Las claves de la optimización de la perforación de pozos (Hankins, 2015; Portilla, 2013; Breyholtz, 2012; MedinaRodríguez, 2012; Kentli, 2009; Onwubolu, 2004; Saputelli, 2003; Van Oort, 2001; Kolahan, 1996; Gray, 1989) son:

\section{- Equipo de trabajo con personal de experiencia}

Los requisitos licitatorios para los servicios de perforación incluyen una sección de personal con los requerimientos técnicos adecuados: se solicitan los currículos detallados del personal asignado al proyecto, organigramas de las compañías oferentes y experiencia en proyectos previos. Una vez completo el equipo, de coordinación en oficina y operativo en campo, se lo mantiene durante toda la campaña de perforación.

\section{- Planificación: Proveedores, Logística, Manejo del} Cambio, Mejora Continua

Cada pozo a ser perforado es planificado con el tiempo adecuado acorde a su complejidad, considerando aspectos técnicos, logísticos y comunicación efectiva entre los miembros del equipo. Como parte de los procesos de mejora continua se cuenta con la herramienta de manejo de cambios que puede ser usada en todo momento para optimizar o cambiar alguna actividad especificada en el programa de perforación.

\section{- Gestión de riesgos: Matriz de riesgos detallada}

La matriz de riesgo es preparada en equipo por los líderes de los servicios involucrados y ajustado por pozo a sus particularidades. Diariamente, y en base a la matriz de riesgos y operaciones del día, se prepara el reporte "Look Ahead" (Schlumberger) donde se colocan los riesgos, planes de contingencia y mitigación y operaciones para las próximas 24 hrs; difundido con el reporte de las 16hrs.

\section{- Integridad Operacional}

Reuniones diarias internas en pozo y en conjunto con los coordinadores de servicios y La Empresa Operadora en oficina, reuniones pre y post operativas, permisos de trabajo, paso a paso, estándares operacionales y la implementación de las mejores prácticas y lecciones aprendidas son claves en el mantenimiento de la integridad operacional.

\section{- Ejecución de las actividades}

Programadas y ejecutadas en tiempo y forma de acuerdo a la planificación.

\section{- Optimización de parámetros}

Un Mapa de Parámetros de perforación actualizado por formación y pozo es preparado e integrado al programa de perforación, preparado usando la experiencia en los pozos previos y los retos en el pozo a perforarse.

\section{- $\quad$ Simuladores de última generación}

Tanto en las etapas de planificación como de ejecución se cuenta con simuladores de última generación para temas de hidráulica, limpieza de hoyo, diseño de brocas, velocidades de viajes, torque y arrastre, etc.

\section{- Tecnologías de punta}

Rotación continua, brocas PDC con insertos de última generación cónicos y de cincel, herramientas de registros mientras se perfora densidad-neutrón sin fuente radioactiva, gamma ray, caliper inducido, resistividad inductiva y resistividad laterolog (imágenes resistivas), fluidos de perforación aditivados para minimizar el daño a la formación y reducir el torque (lubricante líquido y mecánico), estabilizar zonas inestables, reducir torque, minimizar desgaste de tubulares, microscopio digital de alta resolución y espectrómetro de masas (en pozo), entre los principales.

\section{- Prácticas de perforación}

Monitoreo y análisis constante de parámetros para optimizar la limpieza de hoyo, bombeo de píldoras, rotación continua, repasos, toma de surveys optimizados, paradas de circulación acorde a la limpieza del hoyo, etc.

\section{El éxito operativo consiste en:}

- Realizar las operaciones sin afectación a las personas, integridad operacional ni al ambiente.

- Lograr sinergia entre personal de experiencia de las compañías de servicio y operadora trabajando en equipo.

- Estar abiertos a ser progresistas esto es cambio y mejora continua de las actividades respaldados por los análisis de riesgos y viabilidad que correspondan sin tomar atajos.

- Mantener una comunicación clara, concisa y precisa de lo que pasa en el pozo con un plan de comunicación detallado y difundido al personal.

- Realizar un análisis al final de cada pozo para documentar los eventos de calidad, lecciones aprendidas y oportunidades de mejora, a ser implementadas en las operaciones siguientes en lo que aplique.

\subsection{Optimización de Tiempos y Costos}

La optimización de tiempos y costos de las actividades de perforación con el taladro de $1000 \mathrm{HP}$, sin afectar a las personas, integridad operacional ni al ambiente son parte de los pilares para la viabilidad de este tipo de taladros.

En la sección 2.1 se indicaron las principales claves para la optimización de la perforación, esto trae como valor agregado la mejora de los tiempos y los costos asociados a la perforación, cuyo análisis da una retroalimentación que permite a su vez seguir optimizando las operaciones, siendo un circulo virtuoso de mejora continua. Los indicadores considerados fueron los tiempos y costos de la perforación referenciados a los valores estimados de los pozos, comparadas entre sí para un mismo pozo y entre los diferentes pozos de la campaña. 
Previo a la perforación de un pozo se realiza un estimado de tiempos y costos de las actividades de perforación planificadas referenciados a la profundidad del pozo, expresado a través de las curvas de tiempos y costos versus profundidad. Al final de la perforación del pozo se comparan estos tiempos con los reales.

El análisis de los tiempos tanto operacionales como planos, productivos y no productivos son la clave para encontrar las lecciones aprendidas, oportunidades de mejora y optimización de procesos o tareas específicas.

Durante la perforación de un pozo se identifican diferentes tipos de tiempos:

- Tiempos Operacionales, Reales o totales: tiempo real empleado en realizar una actividad durante la perforación.

- Tiempos Limpios: es el tiempo real menos el tiempo no productivo (NPT, non productive time, por sus siglas en ingles).

- Tiempos No Productivos: son tiempos ocupados en solucionar problemas relacionados a la perforación.

- Tiempos Productivos: son los tiempos utilizados en operaciones relacionadas a la perforación.

- Límite técnico: es el tiempo limpio menos los tiempos invisibles, indica cuál es el menor tiempo técnicamente posible para la perforación de un pozo con las condiciones actuales de perforación. Este puede modificarse con la implementación de mejoras en los procedimientos, prácticas y/o tecnologías que se implementen. Donde los tiempos invisibles son los tiempos en exceso (no optimizados) en las operaciones en referencia a los tiempos planificados por actividad.

- Tiempos planos: son los tiempos transcurrido en actividades sin avance en profundidad

Los análisis de los diferentes tiempos de perforación mencionados son mostrados en la sección de resultados.

\subsection{Metodología Empleada}

La metodología empleada en el estudio fue una investigación exploratoria, con un enfoque mixto (cualitativo y cuantitativo); con:

- Recopilar y validar la información de las campañas de perforación de La Empresa Operadora en Ecuador desde el año 2011 en base a los reportes finales de perforación y apoyándose en herramientas informáticas para el manejo de los datos.

- Realizar un análisis comparativo de los tiempos operativos y costos asociados de los taladros utilizados por la Empresa Operadora en la perforación de pozos en Ecuador visualizando las ventajas y desventajas respecto a un taladro de 1000 HP.
- Generar una matriz de riesgos de las operaciones de perforación con barreras (medidas de prevención) claramente identificadas, que eviten factores causales de escalamiento y la materialización de eventos no deseados que afecten a las personas, las operaciones o el medio ambiente ha sido primordial para lograr la viabilidad de usar un taladro de menores capacidades. En complemento la matriz de riesgos debe contar con medidas de control (mitigación) en caso de materializarse un riesgo.

- Realizar un análisis de riesgos para el taladro de perforación que nos permita identificar de forma oportuna los riesgos asociados a su operación y establecer todas las acciones de prevención y mitigación que ameriten para minimizar los riesgos al valor más bajo posible.

- Identificar las oportunidades de mejora en gestión logística, contractual, servicios, operativas (prácticas operacionales, tiempos y costos) con la filosofía GanarGanar entre la operadora y la compañía de servicio que permitan una retroalimentación y mejora continua de los procesos asociados a la perforación de pozos.

- Buscar e implementar las mejores opciones en tecnología, para viabilizar el uso del taladro de 1000 HP sin afectaciones a las personas, integridad operacional ni al ambiente.

- Realizar las perforaciones de pozos con el taladro de 1000 HP en base a la planificación realizada considerando el análisis previo.

- Analizar los resultados

- Generar conclusiones y recomendaciones.

\subsubsection{Metodología para realizar la Matriz de Riesgos de Operaciones de Perforación}

La Matriz de Riesgos de las Operaciones de Perforación es la clave para superar las limitaciones del taladro de 1000 HP y colocar las barreras necesarias para perforar pozos que convencionalmente se perforan con taladros de $2000 \mathrm{HP}$ en la Cuenca Oriente del Ecuador.

El procedimiento de elaboración de la matriz de riesgos consiste en:

- Con los líderes de los servicios de perforación realizar reunión informativa de los datos y alcance del pozo a perforarse y las especificaciones del taladro a usarse.

- Socializar con el personal que ejecuta los servicios en pozo el alcance de la matriz de riesgos a prepararse.

- Identificar y preparar un listado de actividades y riesgos asociados por servicio incluyendo medidas de prevención, mitigación, probabilidad de ocurrencia y una asignación de responsabilidades (Matriz RACI: ¿Quién rinde cuentas? ¿Quién Ejecuta? ¿Quién Asesora? ¿A quién se informa?)

- Realizar el armado de la información de las líneas de servicio en la matriz de riesgos en base al formato de las tablas $2 \mathrm{a}$ y $2 \mathrm{~b}$.

Tabla 2a. Formato de la Matriz de Riesgos de Perforación - Parte 1. Riesgo Inherente

\begin{tabular}{|c|c|c|c|c|c|c|}
\hline SECCIÓN & $\begin{array}{c}\text { LINEA DE } \\
\text { SERVICIO }\end{array}$ & OPERACIÓN & $\begin{array}{c}\text { RIESGOS } \\
\text { INHERENTES }\end{array}$ & PROBABILIDAD & SEVERIDAD & $\begin{array}{c}\text { VALORACIÓN DEL RIESGO } \\
\text { INHERENTE }\end{array}$ \\
\hline
\end{tabular}


Tabla 2b. Formato de la Matriz de Riesgos de Perforación - Parte 2. Riesgo Remanente y RACI

\begin{tabular}{|c|c|c|c|c|c|}
\hline ACCIONES & FACTORES DE ESCALAMIENTO & AVANCE & PROBABILIDAD & SERVERIDAD & RIESGO \\
CORRECTIVAS & PLAN DE ACCIÓN & MATRIZ & RACI \\
\hline
\end{tabular}

- Realizar reunión de revisión y validación de la Matriz con todos los líderes de servicios. Cada servicio debe conocer la información ingresado por los otros ya que sus acciones $\mathrm{u}$ omisiones los pueden afectar.

- Revisión y aprobación de la Operadora

- Difusión entre el personal de oficina y campo de la Matriz de Riesgos

- Retroalimentación y actualización de la matriz de acuerdo a las particularidades del pozo a perforarse y aprobada por los líderes de servicio y la Operadora.

\subsubsection{Metodología para Generar el Análisis de Riesgos del Taladro de $1000 \mathrm{HP}$}

El análisis de riesgos del taladro de perforación requiere un desarrollo particular y complementario a la Matriz de Riesgos de Perforación, enfocado directamente en las limitaciones de los diferentes sistemas del taladro; el proceso consiste en:
- Reunir la información pertinente del taladro y del pozo a ser perforado.

- Realizar un dimensionamiento del taladro requerido para perforar el pozo, estableciendo claramente los requerimientos técnicos.

- Comparar estos requerimientos con los datos técnicos del taladro de $1000 \mathrm{HP}$ y establecer las falencias de este y posibles riesgos.

- Determinar en equipo con los líderes de la compañía proveedora del taladro de perforación, líderes de servicios y la Operadora las medidas de prevención, mitigación y planes de acción que correspondan.

Completar de forma detallada las tablas $3 \mathrm{a}$ y $3 \mathrm{~b}$.

Tabla 3a. Formato del Análisis de Riesgos del Taladro de 1000 HP - Parte 1. Riesgo Inicial

\begin{tabular}{|c|c|c|c|c|c|}
\hline \multirow[b]{2}{*}{$\begin{array}{l}\text { Actividades / } \\
\text { Pasos }\end{array}$} & \multicolumn{2}{|l|}{ PELIGRO } & \multicolumn{3}{|c|}{ RIESGO INICIAL } \\
\hline & $\begin{array}{c}\text { Descripción del Peligro y peores Consecuencias sin medidas de Prevención } \\
\text { o Mitigación en sitio }\end{array}$ & $\begin{array}{l}\text { Categoría de la Perdida / Población } \\
\text { Afectada }\end{array}$ & 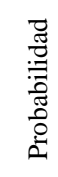 & 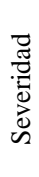 & 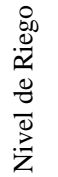 \\
\hline
\end{tabular}

(Fuente: Servicios Integrados de Perforación, Schlumberger, 2018)

Tabla 3b. Formato del Análisis de Riesgos del Taladro de 1000 HP - Parte 2. Riesgo Residual

\begin{tabular}{|c|c|c|c|c|c|c|c|}
\hline \multirow{3}{*}{$\begin{array}{l}\text { Actividades / } \\
\text { Pasos }\end{array}$} & \multicolumn{2}{|c|}{ MEDIDAS DE CONTROL } & \multicolumn{3}{|c|}{ RIESGO RESIDUAL } & \multirow{3}{*}{ 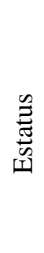 } & \\
\hline & \multicolumn{2}{|c|}{$\begin{array}{c}\text { Colocar todas las Medidas de Control Actuales y Planificadas, tomando en cuenta todos los Factores } \\
\text { que Contribuyen y Agravan }\end{array}$} & \multirow{2}{*}{ 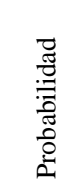 } & \multirow{2}{*}{ 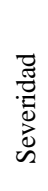 } & \multirow{2}{*}{ 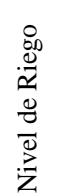 } & & : \\
\hline & $\begin{array}{l}\text { Medidas de Prevención Actuales y Planeadas para } \\
\text { reducir la Probabilidad }\end{array}$ & $\begin{array}{l}\text { Medidas de Mitigación Actuales y Planeadas } \\
\text { para reducir la Severidad }\end{array}$ & & & & & 这 \\
\hline
\end{tabular}

(Fuente: Servicios Integrados de Perforación, Schlumberger, 2018)

- Realizar reunión de revisión y validación del análisis de riesgos del taladro con todos los líderes de servicios. Cada servicio debe conocer la información ingresado; sus acciones u omisiones pueden afectar el desempeño de sus servicios con el taladro.

- Revisión y aprobación la Operadora.

- Difusión entre el personal de oficina y campo del análisis de riesgos del taladro.

- Retroalimentación y actualización del análisis de riesgos del taladro de acuerdo con el rendimiento en pozos previos, a los problemas, mejoras o cambios realizados en el taladro y a las particularidades del pozo a perforarse.

El plan de acción que se genere debe cumplirse en tiempo y forma para evitar eventos que afecten la integridad operacional

\subsubsection{Metodología para Implementar Nueva Tecnología}

La búsqueda y aplicación de las nuevas tecnologías disponibles para la perforación de pozos, que sean apropiadas con el taladro y las operaciones de perforación del presente trabajo sigue los siguientes pasos:

- Realizar la Matriz de Riesgos de las operaciones del pozo a perforarse

- Efectuar el Análisis de Riesgos del taladro

- Buscar las oportunidades de implementar programas especializados de ingeniería, materiales, productos, servicios y/o equipos que sean apropiados de acuerdo con los riesgos y planes de acción preparado.

- Validar las opciones seleccionadas de tal forma que agreguen valor a las actividades y disminuyan los riesgos asociados a las mismas. La tecnología debe ser costo eficiente y estar disponible en tiempo y forma para su 
implementación en la perforación. Esta validación debe realizarse tanto con la compañía proveedora del taladro como las de servicios de perforación para evitar problemas en la implementación o afectaciones negativas en rendimiento, tiempo y/o costo de algún servicio.

Las tecnologías validadas deben ser aprobadas por la operadora e ingresadas al programa de perforación incorporando en el mismo los procedimientos y recomendaciones que correspondan para su correcta implementación.

Posterior a la terminación del pozo se debe evaluar el cumplimiento o no de la tecnología para continuar con su uso, mejorarla o desecharla.

\section{RESULTADOS}

Desde el año 2011 al 2016, la Empresa Operadora de los campos en la Cuenca Oriente del Ecuador, donde se realizó el estudio, perforó 22 pozos con taladros desde 1500 HP hasta $2000 \mathrm{HP}$.

Con el taladro de 1000 HP \# 102 se perforaron entre el 2016 y 2017 un total de 4 pozos, bajo la modalidad de Servicios Integrados de Perforación.

En este trabajo se designaron los pozos como Piloto 01, Piloto 02, Piloto 03 y Piloto 04 (en orden cronológico de perforación).

No se encontraron referencias previas de perforaciones de pozos de las características de los perforados por la Empresa Operadora realizadas con un taladro de $1000 \mathrm{HP}$.

\subsection{Información de Campañas de Perforación}

En la tabla 4 una comparativa de los pozos perforados por La Empresa Operadora desde el 2011 al 2017 con los taladros de 1000 HP, 1500 HP y 2000 HP.

Tabla 4. Comparativo de Costos y Tiempos de los Pozos perforados por La Empresa Operadora del 2011 al 2017 con los taladros de 1000 HP, 1500 HP y 2000 HP.

Promedios

\begin{tabular}{|c|c|c|c|c|c|c|c|c|}
\hline Campaña & Revestidores & \# de Pozos & Torre & Costos (USD) & Tiempo (Días) & Pies perforados (pie) & Costo (USD /pie) & Días /100 pies \\
\hline $2011-2013$ & $\begin{array}{l}3 \text { (13 3/8", } \\
95 / 8 ", 7 ")\end{array}$ & 8 & 1500 a $2000 \mathrm{HP}$ & $5,049,681$ & 23.9 & 10300 & 490.28 & 0.23 \\
\hline 2014-2017 & $\begin{array}{l}2 \text { (13 3/8", } \\
95 / 8 ", 7 ")\end{array}$ & 14 & 2000 & $3,815,659$ & 19.2 & 10629 & 358.97 & 0.18 \\
\hline $2016-2017$ & 2 (9 5/8", 7") & 4 & $1000 \mathrm{HP}$ & $2,991,423$ & 22.2 & 10733 & 278.71 & 0.21 \\
\hline
\end{tabular}

(Fuente: Datos: La Empresa Operadora, 2018; Elaborado por Rodolfo Landivar, 2018)

\subsection{Gestión de Riesgos}

Previa perforación de los pozos se preparó una matriz de riesgos de las operaciones de perforación que se incluyó en los programas de perforación, con mejoras continuas de acuerdo a las lecciones aprendidas en pozos previos, siendo la del pozo Piloto 04 la más actualizada (LA EMPRESA OPERADORA, Programa de Perforación del pozo Piloto 04, 2017g).

Esta matriz presenta en forma detallada los riesgos de la perforación identificados para el escenario de este artículo.

De manera similar se realizó un dimensionamiento del taladro para cada pozo específico.
En base a estos resultados se determinaron los requerimientos del taladro y se los compara con su real capacidad para cada pozo específico. Cabe mencionar que como mejor práctica de la industria los cálculos de dimensionamiento de taladro consideran una sobretensión de $100000 \mathrm{lbs}$, pero por las limitaciones del taladro se consideró el límite de 50000 lbs de sobretensión (overpull por su nombre en inglés) para los cálculos del taladro en todos los casos.

En la tabla 5 se realizó un comparativo de los requerimientos en cuanto a capacidad del taladro de los pozos perforados versus la capacidad del taladro complementado por un sumario de las principales barreras incorporadas. 
Tabla 5. Comparativo de requerimientos del taladro versus su capacidad

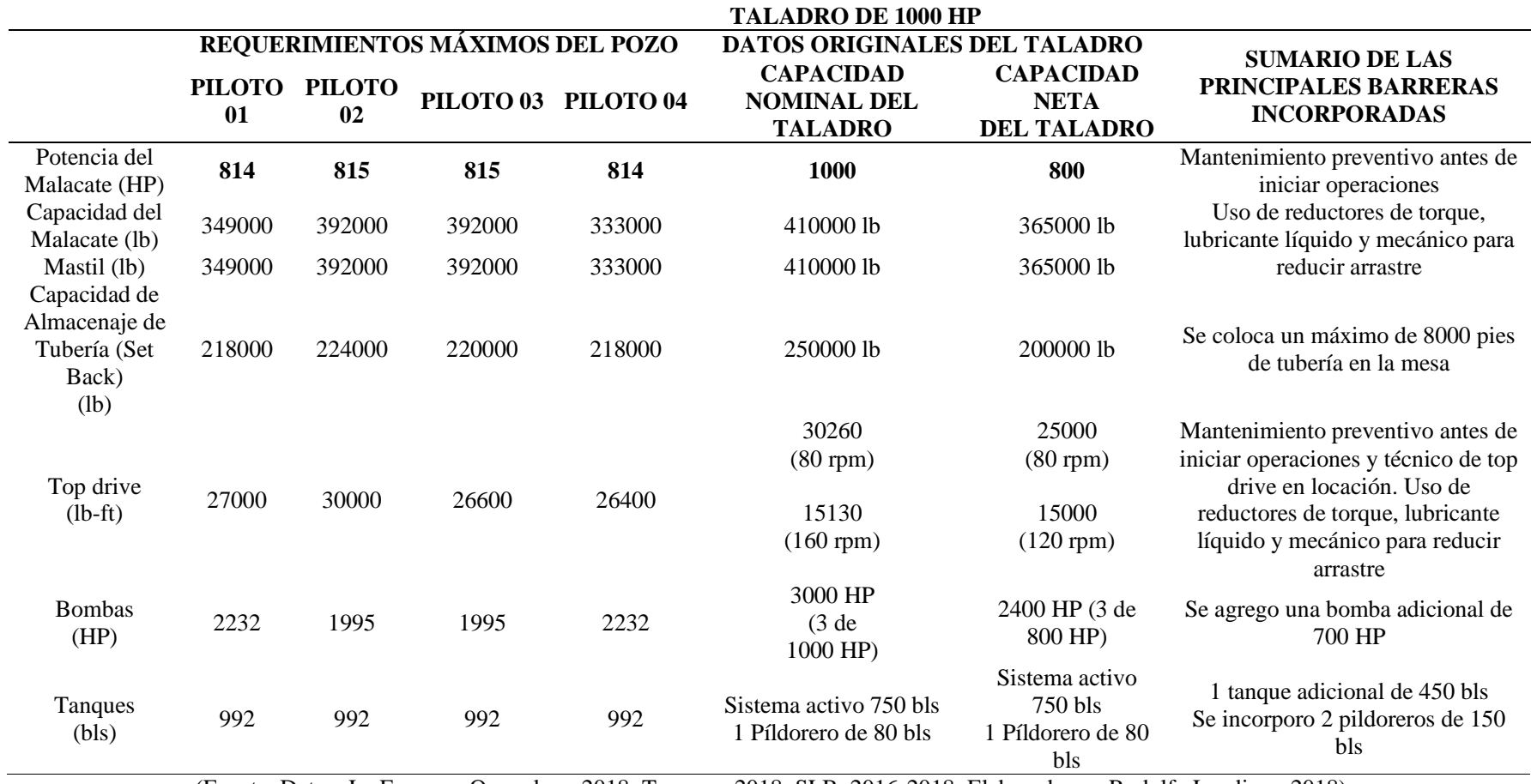

(Fuente: Datos: La Empresa Operadora, 2018; Tuscany, 2018; SLB, 2016-2018; Elaborado por Rodolfo Landivar, 2018)

Previo a la perforación de cada pozo se realizó el análisis de riesgos del taladro de perforación para sus condiciones específicas del mismo tomando en cuenta lo aprendido en los pozos previos, dando paso a un proceso de mejora continua para llevar al valor más bajo posible los riesgos asociados a la perforación.

\subsection{Aplicaciones Tecnológicas y Mejores Prácticas}

Resultado de los datos técnicos del taladro, matriz de riesgos de perforación y del análisis de riesgos del taladro se generaron planes de optimización que fueron mejorándose pozo a pozo.

De las lecciones aprendidas, prácticas operacionales, optimizaciones e implementaciones de tecnología se realizaron implementaciones que agregan valor tanto a las operaciones de perforación como de tiempos planos, a continuación un sumario de los principales aspectos de la perforación en donde se los ha implementado en la campaña de perforación con el taladro de $1000 \mathrm{HP}$, haciendo la diferencia para dar viabilidad al uso de este taladro, destacándose además los beneficios asociados (LA EMPRESA OPERADORA, Programa de Perforación del pozo Piloto 01, 2016a; LA EMPRESA OPERADORA, Programa de Perforación del pozo Piloto 02, 2017c; LA EMPRESA OPERADORA, Programa de Perforación del pozo Piloto 03, 2017e; LA EMPRESA OPERADORA, Programa de Perforación del pozo Piloto 04, 2017g):

- $\quad$ Monitoreo de Torque \& Arrastre (T\&D)

- Reporte de Retorno de Píldoras

- Velocidad de Penetración
- Viajes de Calibración

- Toma de Surveys (Medida de inclinación y azimut)

- Información de Pozo

- Cementación

- Tiempos Planos

- Limpieza de Hoyo

- Repasos

La tecnología aplicada en los pozos estudiados se presenta en la tabla 6 con un sumario de las mismas y la diferencia con los pozos convencionalmente perforados en la cuenca Oriente.

La tabla 6 también presenta un comparativo entre las tecnologías usadas convencionalmente en la Cuenca Oriente del Ecuador y las utilizadas en los pozos perforados con el taladro de $1000 \mathrm{HP}$.

Las principales ventajas se resumen en:

- Mejora en ROP y Optimización de tiempos de exposición de las formaciones perforadas.

- Mejor caliper del hoyo perforado

- Menor número de ensamblajes de fondo

- Buena calidad de los trabajos de cementación

- Obtención de información de pozo

- Optimización de parámetros de perforación

- Mejora del análisis de muestras

- Maximización de la vida útil del ensamblaje de fondo (broca, estabilizadores, patines del motor/RSS).

- Minimizar el volumen de ripios transportados y tratados. 
Tabla 6. Tecnologías aplicadas en los pozos con Taladro de $1000 \mathrm{HP}$

\begin{tabular}{|c|c|c|c|}
\hline Servicios & $\begin{array}{c}\text { Tecnología Aplicada en los pozos } \\
\text { perforados con el taladro de } 1000 \mathrm{HP}\end{array}$ & Pozos de la Cuenca Oriente & $\begin{array}{c}\text { Ventajas diseño aplicado con el } \\
\text { taladro de } 1000 \mathrm{HP} \\
\end{array}$ \\
\hline Perforación Direccional & $\begin{array}{l}\text { Uso de Tecnología Rotary Steereable } \\
\text { (RSS, Power Drive X6 ®SLB) }\end{array}$ & Motores de Fondo & $\begin{array}{l}\text { Mejores ROP, optimización de tiempos } \\
\text { de exposición y daño de formación. } \\
\text { Minimización de washouts y por ende } \\
\text { mejores viajes y cementaciones. } \\
\text { Minimiza riesgos }\end{array}$ \\
\hline Brocas & $\begin{array}{c}\text { Tecnología de punta SteamBlade y Axe } \\
\text { Blade®SLB. Programas IDEAS® SLB, } \\
\text { Análisis DBOS } ® S L B\end{array}$ & Brocas PDC Convencionales & $\begin{array}{l}\text { Mejores ROP. Menor número de BHA. } \\
\text { Optimización de tiempos }\end{array}$ \\
\hline Cementación & $\begin{array}{l}\text { Preflujos mejorados y lechada Optimizada } \\
\text { para mejorar adherencia y control de agua y } \\
\text { gas. Programas CEMANTICS@SLB }\end{array}$ & Lechadas y preflujos convencionales & $\begin{array}{l}\text { Buenas cementaciones. Minimización de } \\
\text { daño a la formación }\end{array}$ \\
\hline Registros LWD & $\begin{array}{c}\text { De Ultima Tecnología (Triple combo sin } \\
\text { fuente e imágenes). NEOSCOPE®SLB - } \\
\text { GVR®SLB }\end{array}$ & No se usa, solo para casos especiales & $\begin{array}{c}\text { Se asegura la información del pozo y } \\
\text { minimiza tiempos de exposición de las } \\
\text { formaciones productoras, reduciendo el } \\
\text { daño de formación }\end{array}$ \\
\hline Fluido de Perforación & $\begin{array}{l}\text { Uso de lubricante líquido (Hydraspeed } \\
\text { ®SLB) y mecánico (Drillbeads ®SLB), } \\
\text { puente particular por pozo y formación. } \\
\text { Software Virtual Hydraulics®SLB } \\
\text { Equipo de especial. Ripios con menos del }\end{array}$ & Fluidos convencionales & $\begin{array}{l}\text { Minimización del daño de formación y } \\
\text { optimización de parámetros de } \\
\text { perforación por la disminución del torque } \\
\text { y arrastre. Minimiza riesgos }\end{array}$ \\
\hline Control de Solidos & $\begin{array}{l}40 \% \text { de humedad. Uso de zaranda secadora. } \\
\text { Utilización de unidad recuperadora de } \\
\text { Drillbeads (lubricante mecánico) }{ }^{\circledR S L B} \\
\text { Uso de equipos de punta como el }\end{array}$ & Equipo de control convencional & $\begin{array}{l}\text { Minimiza volumen de ripios a ser } \\
\text { transportados y tratados. Menos viajes. }\end{array}$ \\
\hline Mud Logging & $\begin{array}{c}\text { DQ1000®SLB (Espectrógrafo de Masas) y } \\
\text { Microscopio Digital de alta resolución directo } \\
\text { en pozo }\end{array}$ & Equipo de monitoreo convencional & $\begin{array}{l}\text { Mejora del análisis de muestras de pozo } \\
\text { que apoyan la decisión operativa }\end{array}$ \\
\hline BHA & $\begin{array}{l}\text { Uso de Reductores de torque para maximizar } \\
\text { la vida útil del bha }\end{array}$ & Sarta convencional & $\begin{array}{c}\text { Mejora vida del ensamblaje y trabajo del } \\
\text { taladro. Optimiza parámetros de } \\
\text { perforación }\end{array}$ \\
\hline
\end{tabular}

(Fuente: Elaborado por Rodolfo Landivar, 2018)

\subsection{Resultados de la Campaña de perforación con el taladro de $1000 \mathrm{HP}$}

Los resultados de los 4 pozos perforados con el taladro de 1000 HP del 2016 al 2017 están basados en los datos de los reportes finales de perforación (LA EMPRESA OPERADORA, Reporte Final de Perforación del pozo Piloto 01, 2016b; LA EMPRESA OPERADORA, Reporte Final de Perforación del pozo Piloto 02, 2017d; LA EMPRESA OPERADORA, Reporte Final de Perforación del pozo Piloto 03, 2017f; LA EMPRESA OPERADORA, Reporte Final de Perforación del pozo Piloto 04, 2017) y se presentan a continuación:

Los pozos perforados fueron direccionales tipo "J" Modificado en dos secciones (12 1/4" y 8 1/2") y completados con un revestidor superficial de $95 / 8$ " asentado al tope de la formación Tiyuyacu y un liner de 7" asentado en la formación
Hollín. El diseño de los revestidores consideró que ambos eran de producción por lo que se les aplicó el caso de evacuación total.

No se presentaron eventos de pérdidas de equipos o herramientas en el pozo, terminando todos los pozos a las profundidades programadas.

Se mantuvo las mismas compañías de servicio durante todo el proyecto, así como al personal involucrado en las operaciones. Los tiempos y costos reales de perforación de los pozos se mantuvieron por debajo de lo estimado.

En tabla 7 los tiempos, costos y profundidades alcanzadas por pozo con indicadores de costo por pie y días por cada 100 pies perforados, con la finalidad de disponer de parámetros de comparación.

Tabla 7. Indicadores de los pozos perforados por el taladro de $1000 \mathrm{HP}$

\begin{tabular}{|c|c|c|c|c|c|c|c|}
\hline Pozo & Revestidores & Torre & Costos (USD) & Tiempo (Días) & Pies perforados & Costo por pies (USD/pie) & Días /100 pies \\
\hline Piloto 01 & \multirow{5}{*}{$\begin{array}{c}2 \\
(95 / 8 ", 7 ")\end{array}$} & \multirow{4}{*}{$1000 \mathrm{HP}$} & $2,895,017$ & 23.4 & 10882 & 266 & 0.22 \\
\hline Piloto 02 & & & $2,959,590$ & 22.0 & 10665 & 278 & 0.21 \\
\hline Piloto 03 & & & $2,999,107$ & 20.4 & 10808 & 277 & 0.19 \\
\hline Piloto 04 & & & $3,111,979$ & 23.2 & 10578 & 294 & 0.22 \\
\hline & & Promedio & $2,991,423$ & 22.2 & 10733 & 279 & 0.21 \\
\hline
\end{tabular}

Para el pozo Piloto 01 se estimaron los tiempos de perforación considerando el peor escenario respecto a tiempos, incluyendo un viaje de cambio de ensamblaje de fondo en las secciones de 12 1/4" y 8 1/2" dando un total de 26.5 días. Para el resto de los pozos se decidió mantener el tiempo planificado para disponer un valor de referencia y comparar de forma objetiva el tiempo planeado y los reales de los cuatro pozos perforados.

Las curvas de tiempos de perforación real versus profundidad de los 4 pozos perforados se muestran en la figura 1, incluyendo los datos de adelanto en días respecto al estimado 
de tiempo de perforación planificado que en todos los casos se consideró 26.5 días, que era el tiempo considerado en el peor escenario.

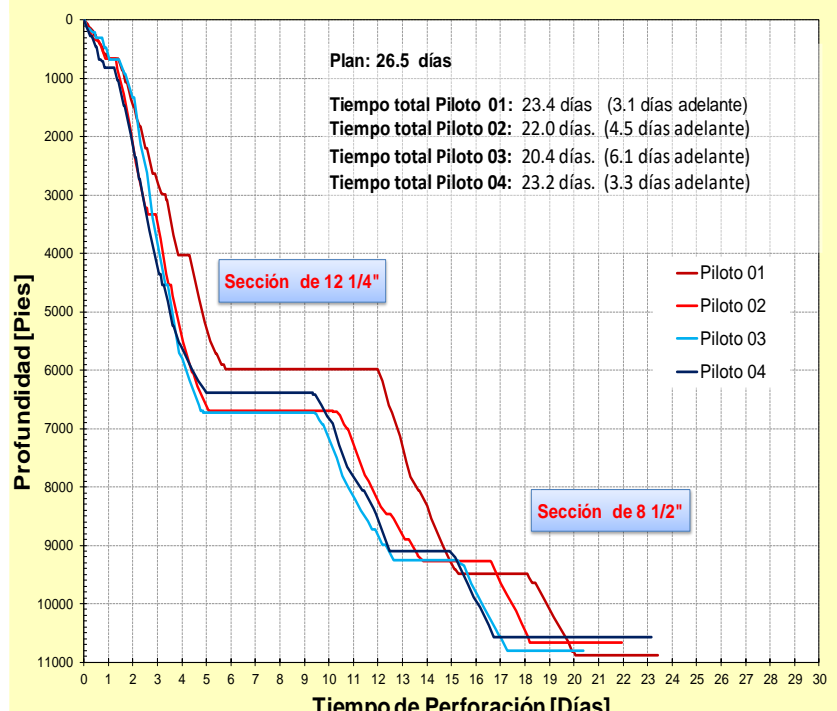

Figura 1. Curvas de Tiempos vs Profundidad de los pozos perforados (Fuente: La Empresa Operadora, 2018)

El análisis de la información es crucial para encontrar puntos de mejora, cambiar procesos o implementar nuevos que agreguen valor minimizando los tiempos y bajando los costos de la operación. En las figuras 2 y 3 se muestra el rendimiento general por pozo.

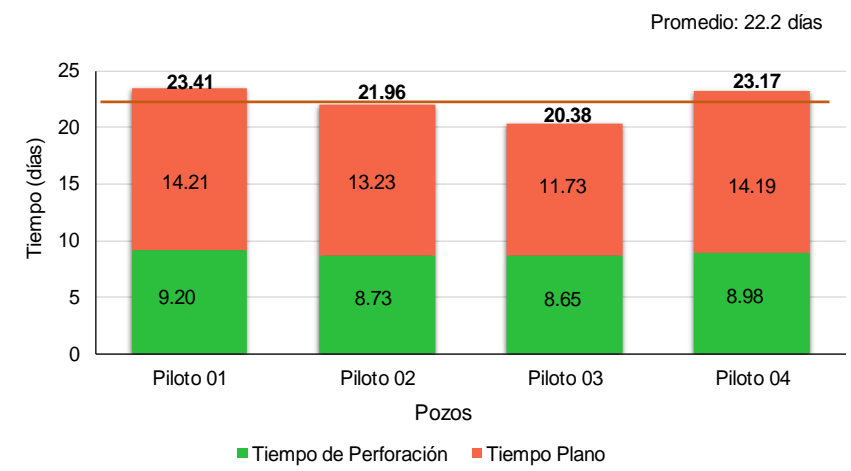

Figura 2. Rendimiento general de los pozos - Tiempos Perforando vs Planos (en días) (Fuente: Datos: La Empresa Operadora, 2018; Programa Righour ®Schlumberger, 2018; Elaborado por Rodolfo Landivar, 2018)

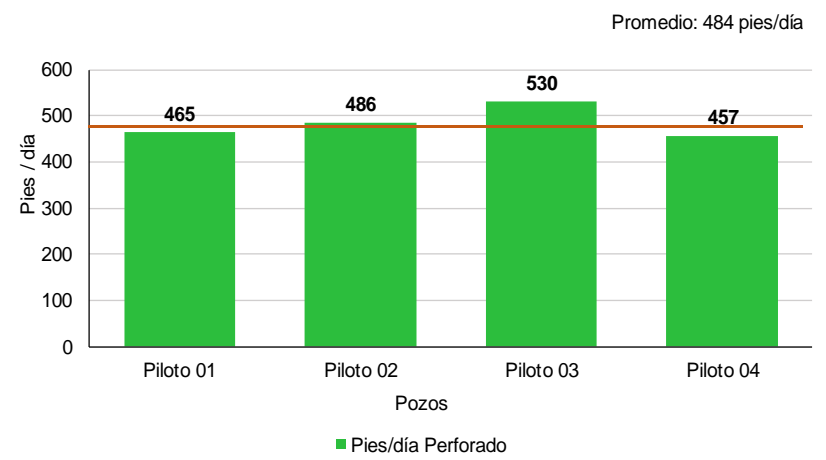

Figura 3. Pies por día perforados por pozo (Fuente: Datos: La Empresa

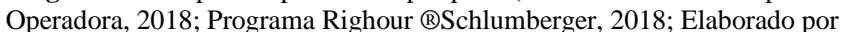
Rodolfo Landivar, 2018)
En los tiempos planos, los de mayor peso son los viajes tanto a superficie como a fondo de los ensamblajes, así como corridas de los revestidores, por lo que se realizó su revisión y evolución de un pozo a otro, verificando de esta forma si el plan de optimización cumplió su meta.

Las figuras 4 y 5 muestran las velocidades de viaje en las secciones de $121 / 4$ " y 8 1/2", respectivamente.

En la sección de 12 1/4" no se observan datos del viaje a fondo debido a que en este hoyo no se lo realiza.



Figura 4. Velocidades Promedio de Viaje en la sección de 12 1/4" (Pies por hora) (Fuente: Datos: La Empresa Operadora, 2018; Elaborado por Rodolfo Landivar, 2018)



Figura 5. Velocidades Promedio de Viaje en la sección de 8 1/2" (Pies por hora) (Fuente: Datos: La Empresa Operadora, 2018; Elaborado por Rodolfo Landivar, 2018)

Otro de los tiempos planos que presentan oportunidades de mejora, son los tiempos de repasos, conexión y registros de desviación (surveys), cuyos resultados son comparados entre los pozos perforados con el taladro de $1000 \mathrm{HP}$; ver figura 6 .

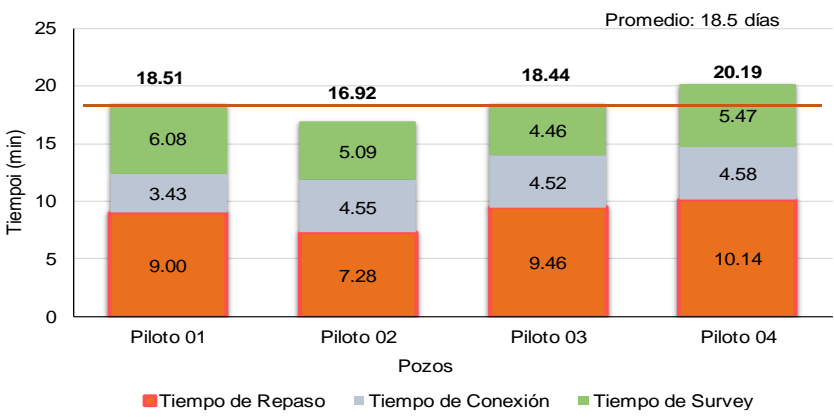

Figura 6. Rendimiento del Taladro en tiempos de repasos, conexión y survey (min por parada) (Fuente: Datos: La Empresa Operadora, 2018; Programa Righour @Schlumberger, 2018; Elaborado por Rodolfo Landivar, 2018) 
En base a los tiempos no productivos, tanto por pozo como por categoría, se generó la figura 7 donde se puede identificar los factores que nos afectan en los tiempos y poder tomar las medidas necesarias para reducir el mismo. Esta información alimentará el análisis de riesgos del taladro y validará las diferentes medidas de prevención tomadas o generará una oportunidad de mejora.

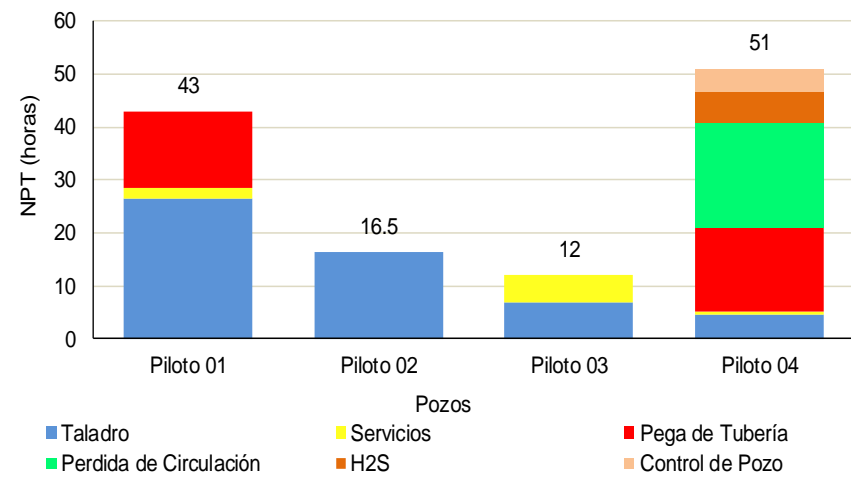

Figura 7. Tiempo no Productivo (NPT) por pozo y por categoría (horas por actividad) (Fuente: Datos: La Empresa Operadora, 2018; Programa Righour (B) Schlumberger, 2018; Elaborado por Rodolfo Landivar, 2018)

En la figura 8 se presentan los comparativos de tiempos de los pozos perforados que permiten identificar el límite técnico promedio de un pozo perforado con un taladro de 1000 HP en 19 días.

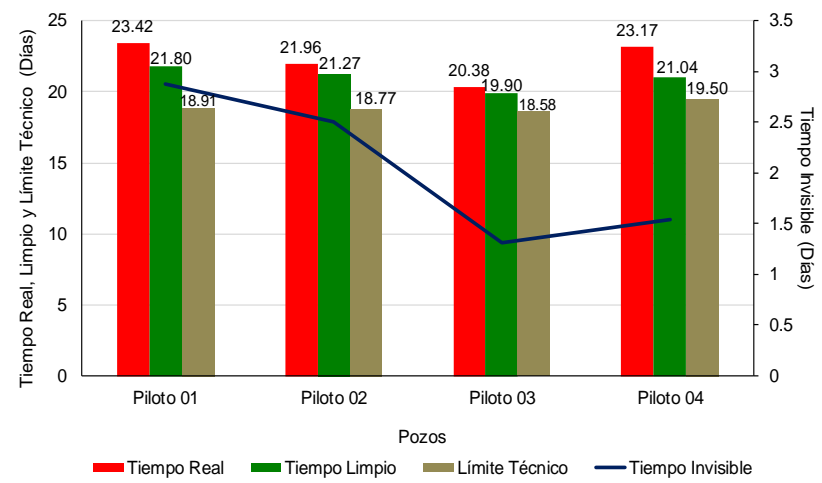

Figura 8. Comparativa de tiempos reales, limpios, límite técnico e invisible (Fuente: Datos: La Empresa Operadora, 2018; Elaborado por Rodolfo Landivar, 2018)

La comparativa de las curvas de costos reales de los pozos perforados se presenta en la figura 9 , mostrando un sumario de los ahorros por pozo.



Figura 9. Curvas de Costos vs Profundidad de los pozos perforados (Fuente: La Empresa Operadora, 2018)

\section{DISCUSIÓN}

De los 4 pozos perforados y analizados, el pozo Piloto 1 llego a la mayor profundidad de $10882 \mathrm{ft}$ con un desplazamiento horizontal de 3282 pies con un Angulo máximo de 24.3 .

La gestión de riesgos realizada con el taladro de 1000 HP en base a los requerimientos de los pozos perforados permitió identificar las falencias, debilidades y oportunidades de mejora del taladro de 1000 HP.

Para viabilizar la perforación de pozos a profundidades promedios de 10500 pies a la formación Hollín con desplazamientos del orden de 3300 pies con un taladro de 1000 HP se realizó un gerenciamiento de los riesgos a través de una matriz de riesgos de las actividades de perforación iniciando en el primer pozo de la campaña, el Piloto 01 (LA EMPRESA OPERADORA, Programa de Perforación del pozo Piloto 01, 2016a) y la respectiva actualización para cada nuevo pozo, complementado con un análisis de riesgos del taladro de tal forma de identificar los riesgos y colocar todas las barreras que se requieran para evitar eventos que afecten a las personas, la integridad operacional y al ambiente.

En la tabla 4 se observa la evolución de pozos de 3 a 2 revestidores con taladro de al menos $1500 \mathrm{HP}$ con ahorros promedios de costos de $\$ 1.2$ Millones de dólares y 4.7 días. Al comparar a su vez el diseño de 2 revestidores realizado con el taladro de $1000 \mathrm{HP}$ con el realizado con el taladro de 2000 $\mathrm{HP}$, se observa una disminución del costo promedio por pozo de 824 mil dólares por pozo a pesar de que los tiempos por pozo se incrementan en 3 días.

En la tabla 7 y figura 1 se observa que del pozo Piloto 01 al Piloto 03 hubo una mejora continua en los tiempos de la perforación de 23.4 días a 20.4 días producto de la implementación continua de procesos de mejora en el taladro como se indica en la tabla 5 acerca de las barreras incorporadas, complementado con el análisis de riesgo, además de la optimización operativa a través de la aplicación de las mejores prácticas de perforación y lecciones aprendidas, realizando sinergia con la tecnología de punta (ver tabla 6) utilizada en los pozos perforados con el taladro de $1000 \mathrm{HP}$.

El pozo Piloto 04 estuvo afectado en tiempos ante la presencia de $\mathrm{H} 2 \mathrm{~S}$, avenidas de gas y eventos de pérdida de circulación en el hoyo de $81 / 2$ " durante la bajada del liner de 7", que fueron anómalos para el campo, ya que de la información de los pozos previos no se tenía referencias similares. En complemento, observando la curva de tiempos versus profundidad del pozo Piloto 04 al final de la perforación (figura 1) se evidencia un adelanto respecto al pozo Piloto 03 que tiene el menor tiempo, mostrando que, si no hubieran ocurrido los eventos mencionados, el Piloto 04 hubiera sido el mejor pozo de la campaña.

El rendimiento por pozo mostrado figura 2, muestra una tendencia a disminuir los tiempos planos 14.2 días en el pozo Piloto 01 a 11.7 días en el pozo Piloto 03, en adición el tiempo perforando baja de 9.2 días a 8.6 días respectivamente. El 
efecto de los eventos en el Piloto 04 afectaron en particular a los tiempos planos llegando a 14.19 días mientras que el tiempo perforando fue de 8.98 días.

En cuanto a las velocidades de viaje (ver figuras 4 y 5 ), los viajes a superficie presentan tendencia a aumentar en las fases de 12 1/4" y 8 1/2", indicando buenas condiciones de hoyo; en particular resalta el pozo Piloto 04, en la sección de 12 1/4" presenta una velocidad de $600 \mathrm{pies} / \mathrm{hr}$.

En la figura 4 no se marcan las velocidades de viaje a fondo debido a que una de las optimizaciones del plan de perforación era optimizar los tiempos de la sección de 12 1/4" eliminando el viaje de calibración. Esta práctica fue muy exitosa y como se observa en ninguno de los 4 pozos se realizó. En la sección de $8 \frac{1}{2} 2$ " (ver figura 5) no se observa mejora en velocidades de viaje a fondo en los dos primeros pozos (Piloto 01 y Piloto 02) pero si en los dos últimos producto de la mejora en las practicas operacionales y la implementación de los lubricantes líquido (Lube y Hydraspeed @SLB) y mecánico (Drillbeads®SLB) en el fluido de perforación, llegando a duplicar la velocidad de bajada de $251 \mathrm{pies} / \mathrm{hr}$ en el Piloto 01 a 550 pies/hr en el Piloto 04.

En cuanto a las velocidades de corrida de revestidor, tanto en las secciones de $12 \frac{1 / 4}{4}$ " como en $8 \frac{1}{2}$ ", se observa una tendencia a aumentar excepto en el pozo Piloto 04, donde el liner de 7" tuvo la menor velocidad de corrida de la campaña con 170 pies/hr atribuyéndose al mayor tiempo de exposición de las formaciones y mayor cantidad de maniobras en el hoyo.

Los tiempos del taladro respecto a repasos (ver figura 6) tuvo tendencia a aumentar desde el inicio de la campaña debido al objetivo de dejar el hoyo en buenas condiciones con el ensamblaje de perforación saliendo y entrando libre, de tal forma que se invirtió tiempo en acondicionar el hoyo.

Para los tiempos de conexión no se observaron mejoras significativas debido principalmente a la aplicación parcial de la llave de tubulares ST61 que no se la pudo tener disponible todo el tiempo. Los tiempos de registro direccional (survey) han presentado un comportamiento irregular con su mejor tiempo en el pozo Piloto 03 con 4.5 min y el peor tiempo en el pozo Piloto 01 con $6.1 \mathrm{~min}$.

Los tiempos no productivos (NPT, No productive time, por sus siglas en inglés) cayeron de 43 hrs en el Piloto 01 a 12 hrs en el Piloto 03 (ver figura 7), principalmente a las acciones preventivas tomadas para gestionar los riesgos debido a las limitaciones de capacidad del taladro de $1000 \mathrm{HP}$ (ver tablas 5). En el pozo Piloto 04, el NPT debido a condiciones de hoyo (H2S, pérdidas de circulación e influjos de gas) causaron que el NPT llegue a $50.7 \mathrm{hrs}$.

La comparativa de tiempos reales, tiempos limpios, límite técnico y tiempos invisibles (ver figura 8) muestra una disminución continua de los mismos desde el Piloto 01 al Piloto 03, cayendo los tiempos limpios desde 21.8 días a 19.9 días, respectivamente. Nuevamente los indicadores del pozo Piloto 04 se ven afectados por los eventos durante la perforación de la fase de $8 \frac{1}{2}$ ”. El límite técnico promedio de un pozo de 2 revestidores (9 5/8" x 7") con un taladro de 1000 HP está en 19 días. Lo anterior se ve corroborado por los pies por día perforados mostrados en la figura 8 que tuvieron una tendencia ascendente hasta llegar a los 530 pies/día en el Piloto 03 cayendo a 457 pies/día en el Piloto 04.

En el tema de costos de los pozos (ver tabla 7 y figura 9), la diferencia de costos es de aproximadamente \$ 104 mil dólares. En el caso del pozo Piloto 04 debido a los eventos mencionados se aleja de los costos de los pozos previos llegando a \$ 3.1 Millones de dólares

Los resultados expuestos muestran que la metodología aplicada permitió la viabilidad del uso del uso de un taladro de $1000 \mathrm{HP}$ en la perforación de pozos que por sus profundidades venían siendo perforados con taladro de $1500 \mathrm{HP}$ o superiores.

\section{CONCLUSIONES}

Las perforaciones de pozos con el taladro de perforación de 1000 HP son viables técnicamente.

Los 4 pozos perforados por la Empresa Operadora en los campos de la Cuenca Oriente del Ecuador se completaron sin afectaciones a las personas, integridad operacional ni al ambiente.

La metodología empleada en el trabajo se validó con los resultados de los pozos perforados y puede ser replicada en otros proyectos.

La gestión de riesgos fue clave para lograr la aplicabilidad del taladro de $1000 \mathrm{HP}$.

El pozo Piloto 01 fue el de mayor profundidad llegando a los 10882 pies de profundidad, con 3282 pies de desplazamiento horizontal y $24.3^{\circ}$ de inclinación, siendo perforado en hoyos de 12 1/4" x 8 1 $\frac{1}{2}$ " y completado con revestidores de $95 / 8$ " y liner de 7', respectivamente.

El límite técnico de un pozo perforado por un taladro de 1000 HP es de 19 días mientras que el promedio de tiempo de perforación actual es de 22.2 días, lo que implica que hay oportunidades de mejora que deben ser aprovechadas con la retroalimentación continua de lecciones aprendidas, mejores prácticas y opciones de nueva tecnología a implementarse en los pozos.

El uso de un taladro de 1000 HP permitió un ahorro de \$ 334 K USD en promedio en los pozos perforados.

Se han perforado los pozos con un promedio de 4.4 días de adelanto relativo al plan de perforación.

Los 4 pozos perforados presentan un porcentaje de producción real versus la estimada de $218 \%$ mostrando que la perforación fue realizada sin afectación a las formaciones productoras.

El logro de perforar pozos a Hollín con un taladro de $1000 \mathrm{HP}$ abre las puertas a la Empresa Operadora de ampliar su 
campaña de perforación y a otros operadores de tomar esta experiencia y aplicarla en sus proyectos.

El trabajo del personal técnico de la Empresa Operadora, del proveedor de taladro perforación, de la compañía de Servicios Integrados y de las demás compañías que brindaron servicios complementarios, han dados resultados positivos creando sinergia y gestionando los riesgos.

\section{RECOMENDACIONES}

Mantener en las próximas campañas de perforación de la Empresa Operadora el uso del taladro de $1000 \mathrm{HP}$ en la Cuenca Oriente del Ecuador y replicar en otros proyectos de la compañía a nivel internacional.

Replicar esta experiencia en otras operadoras agregando valor a sus proyectos con el ahorro de tiempos y costos.

Continuar el proceso de mejora continua para implementar nuevas barreras, lecciones aprendidas, prácticas operacionales y tecnologías para llegar al límite técnico del tiempo de perforación.

\section{AGRADECIMIENTOS}

El autor agradece a la compañía Operadora de los Campos donde se realizó el estudio, a Tuscany Perforación Petrolera Tuscanyperf S.A. ("Tuscany") y Schlumberger del Ecuador S. A. que han permitido usar su información para el desarrollo del trabajo. A la Escuela Politécnica Nacional y en particular a sus Profesores Msc. Marco Loaiza y Msc. Raúl Valencia por el apoyo brindado.

\section{REFERENCIAS}

ARCH Agencia de Regulación y Control Hidrocarburífero. (2015). Boletín Estadístico. Recuperado de http://www.controlhidrocarburos.gob.ec/wpcontent/uploads/boletin-estadistico/ESTADI\%CC\%81STICAS-ARCH2015_2016-07-15_Para-publicar.pdf

Breyholtz, Ø., \& Nikolaou, M. (2012). Drilling automation: Presenting a framework for automated operations. SPE Drilling \& Completion, 27(01), 118-126.

LA EMPRESA OPERADORA. (2016a). Programa de Perforación del pozo Piloto 01

LA EMPRESA OPERADORA. (2016b). Reporte Final de Perforación del pozo Piloto 01

LA EMPRESA OPERADORA. (2017c). Programa de Perforación del pozo - Piloto 02

LA EMPRESA OPERADORA. (2017d). Reporte Final de Perforación del pozo -Piloto 02

LA EMPRESA OPERADORA. (2017e). Programa de Perforación del pozo Piloto 03

LA EMPRESA OPERADORA. (2017f). Reporte Final de Perforación del pozo Piloto 03

LA EMPRESA OPERADORA. (2017g). Programa de Perforación del pozo Piloto 04

LA EMPRESA OPERADORA. (2017h). Reporte Final de Perforación del pozo Piloto 04

LA EMPRESA OPERADORA. Departamento de Perforación. (2016). Comparativa de un Taladro de $1000 \mathrm{HP}$ vs 2000 HP.ppt

Gray, R. L., \& Cambridge, V. J. (1989). U.S. Patent No. 4,845,628. Washington, DC: U.S. Patent and Trademark Office.

Hankins, D., Salehi, S., \& Karbalaei Saleh, F. (2015). An Integrated Approach for Drilling Optimization Using Advanced Drilling Optimizer. Journal of Petroleum Engineering, 2015.
Kentli, A., \& Alkaya, A. F. (2009). Deterministic approach to path optimization problem. Ozean Journal of Applied Sciences, 2(2), 149-157.

Kolahan, F., \& Liang, M. (1996). A tabu search approach to optimization of drilling operations. Computers \& industrial engineering, 31(1-2), 371374.

Medina-Rodríguez, N., Montiel-Ross, O., Sepulveda, R., \& Castillo, O. (2012). Tool path optimization for computer Numerical control machines based on parallel ACO. Engineering Letters, 20(1), 101.

Onwubolu, G. C., \& Clerc, M. (2004). Optimal path for automated drilling operations by a new heuristic approach using particle swarm optimization. International Journal of Production Research, 42(3), $473-$ 491.

Portilla, H. E., Suárez, D. F., \& Corzo, R. (2013). Metodología para la optimización de parámetros de perforación a partir de propiedades geomecánicas. REVISTA FUENTES, 10(2).

Saputelli, L., Economides, M., Nikolaou, M., \& Kelessidis, V. (2003, January). Real-time decision-making for value creation while drilling. In SPE/IADC Middle East Drilling Technology Conference and Exhibition. Society of Petroleum Engineers.

TUSCANY. (2018). RIG 102 SPECS and Inventory Perforacion.pdf

TUSCANY. (2018). RIG 117 SPECS and inventory.pdf

Van Oort, E., Nicholson, J., \& D'Agostino, J. (2001, January). Integrated borehole stability studies: Key to drilling at the technical limit and trouble cost reduction. In SPE/IADC drilling conference. Society of Petroleum Engineers

\section{BIOGRAFÍAS}

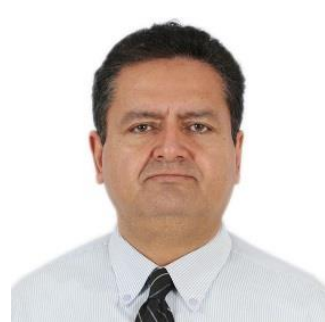

Rodolfo Landívar. Ingeniero en Petróleos de la Escuela Politécnica del Litoral, Master en "Gestión para la Exploración y Producción de Petróleo" de la Escuela Politécnica Nacional, cuenta con un diplomado en Desarrollo Directivo del IDE Business School. Profesional con 27 años de experiencia en la Industria Hidrocarburífera Nacional e Internacional. Ing. de Reservorios y Producción en la DNH, Ing. de Registros Eléctricos en Halliburton, Lider de Ingeniería en B.J. Services, Especialista en Completaciones y Lider del Proyecto de Perforación en Petrobras, Senior en Construcción de Pozos y Gerente de Perforación en Ivanhoe Energy Ecuador, Senior de Perforación y Gerente de Proyectos de Servicios Integrados en Schlumberger del Ecuador S. A. Actualmente es Gerente de Proyectos de Schlumberger asignado a ENAP SIPEC.

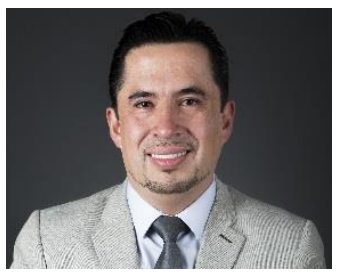

Marco Loaiza Córdova: Ingeniero de Petróleos Escuela Politécnica Nacional. Posee un MBA de la Universidad Francisco de Victoria de Madrid, un Master en Ciencias en Petróleo y Gas por la Swiss Business School, un Master en Política y Estrategia Empresarial por el Tecnológico de Monterrey, 14 años en la industria Nacional e Internacional en Argentina, Brasil \& USA, en Petrobras Ecuador, Petrobras Internacional y Tecpetrol. Actualmente se desempeña como como Líder Senior de Perforación en ENAP SIPEC, además es profesor de la Maestría en Gestión para la Exploración y Producción del Petróleo en la Escuela Politécnica Nacional y también es Docente en Maestría en Petróleos, Mención en Procesos de Producción e Industrialización de Hidrocarburos en la Universidad Tecnológica Equinoccial. 


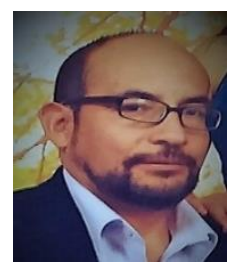

Raúl Valencia. Ingeniero en Petróleos y Magister en Ingeniería Industrial por la Escuela Politécnica Nacional. Profesor Titular a Tiempo completo en la Carrera de Ingeniería en Petróleos desde 1998. Actualmente es jefe del Departamento de Petróleo. Sus intereses de investigación se centran el Área de Petróleos específicamente en la Línea de Yacimientos de Hidrocarburos y Recuperación Mejorada. 